\title{
Editorial
}

\section{Cancer and thrombosis: a fresh look at an old story}

\section{Introduction}

Exactly 150 years ago, a French physician, Armand Trousseau published his findings on the association between gastric cancer and thrombosis [1]. This paper, and that published by Bouillard in 1823, [2] are now recognized as the earliest clinical descriptions on the association between malignant diseases and hemostasis.

In the past decade, hundreds of studies have provided considerable evidence that in patients with cancer, blood coagulation is shifted in the direction of a prothrombotic state. Cancer and its treatments represent well-recognized risk factors for venous thromboembolism (VTE) [3]. Apart from clinical risk factors, such as advanced age, immobility, surgery, and others, there are tumor-related mechanisms contributing to the prothrombotic diathesis in patients with cancer. At least three mechanisms are known by which tumor cells can activate hemostasis: 1 ) by the production of procoagulant, fibrinolytic, and proaggregating factors, such as tissue factor (TF), cancer procoagulant $(\mathrm{CP})$, plasminogen activator inhibitors, ADP, and others; 2 ) by releasing proinflammatory and proangiogenic cytokines, such as tumor necrosis factor $\alpha$ and interleukin $1 \beta$; and 3 ) by directly interacting with host vascular and blood cells, such as endothelial cells, leukocytes, and platelets through adhesion molecules [4]. A large body of work has shown that tumor cells activate blood coagulation through an increased expression of TF [5]. Upregulation of TF in cancer cells may be due to tumor-specific oncogenic transformations or hypoxia. TF expressed by vascular, stromal and inflammatory cells might also contribute to the procoagulant effect of the tumor environment. TF can also be released on TF-exposing extracellular vesicles in the circulation [6]. Although the mechanism is not entirely clear yet, correlation of TF expression with a hypercoagulable state and an increased incidence of VTE has been revealed in experimental models and in certain tumor types, such as pancreatic cancer [6,7]. In addition to constitutive activation of the extrinsic pathway, increased levels of coagulation factors V, VIII, IX and XI have been shown to contribute to the prothrombotic state in patients with cancer [8]. Another extensively studied tumor cell procoagulant is $\mathrm{CP}$, which is a cysteine protease that is capable of activating factor $\mathrm{X}$ independently of factor VII [9]. CP has been found in extracts of neoplastic cells and in cancer patients' sera but not in extracts of normally differentiated cells. In addition to the activation of the coagulation system, cancer cells produce proteins that are involved in the regulation of fibrinolysis. These include the expression of plasminogen activators as well as plasminogen activator inhibitors 1 and 2, leading to an imbalance of fibrinolysis [4]. Tumor cells are also involved in direct cell-cell interactions leading to a prothrombotic condition. This includes the activation of platelets and white blood cells through the release of certain soluble mediators, proteases, or cytokines.
More interestingly, it has been evident for decades that there is a two-way interplay between coagulation and cancer, i.e., the procoagulant environment brought about by malignant cells may also promote cancer progression. Although significant progress has been made in this area, the role of various elements of hemostasis in malignant disease progression and tumor angiogenesis remains to be elucidated. This direction of research is fascinating; in addition to enhancing our understanding of cancer progression and regulation, it could ultimately lead to the discovery of innovative anticancer therapies. In early phases of metastasis, thrombin, generated by TF initiates fibrin deposition and the recruitment of platelets. This process has proven to be important in increasing the adhesive properties and survival of tumor cells [5]. The adhesion of platelets to tumor cells plays a substantial role in tumor growth and metastasis. Inhibition of various mechanisms mediating platelet adhesion to tumor cells results in a significant inhibition of tumor dissemination in experimental animal models [10]. The complex anticoagulant mechanisms of the endothelium, including endothelial cell protein $C$ receptor, thrombomodulin and heparan sulfate proteoglycan, and its impairment may also play a role in tumor cell metastasis by regulating local thrombin levels $[11,12]$. Thrombin signals to endothelial cells and platelets through cleavage of protease activated receptors (PARs). Thus, thrombin affects tumor growth and metastasis by many separate mechanisms: by leading to fibrin formation, acting on platelets and triggering tumor and endothelial cell signaling [13]. In addition to thrombin, a broad range of proteases acting on PARs have emerged as important regulators of tumor progression and metastasis. These proteases, including factor $\mathrm{Xa}$, plasmin, activated protein $\mathrm{C}$, matrix metalloproteinases, tissue kallikreins, and membrane-type serine proteases have been implicated in the regulation of tumor microenvironment and in the cellular crosstalk associated with tumor progression $[5,10]$.

\section{The Role of Fibrinogen-like Protein 2 (FGL-2)/fibroleukin in Tumor Development}

In this issue of Thrombosis Research, Rabidazeh et al. elaborate on the role of the procoagulant protein, fibrinogen-like protein 2 (FGL-2)/ fibroleukin in tumorigenesis [14]. FGL-2, a member of the fibrinogen family of proteins, is a transmembrane prothrombinase exerting serine protease activity that is capable of converting prothrombin to thrombin in the absence of factor VII or factor X [15]. FGL-2 has been shown to be involved in angiogenesis and it was found to be overexpressed in tumor cells $[16,17]$. The exact role of FGL-2 in tumor development however, has not been fully elucidated yet. A recent study found that knockdown of FGL-2 delayed tumor growth and angiogenesis in mice injected with 
human hepatocellular carcinoma cell line [18]. The underlying mechanism was hypothesized to be a result of FGL-2 induced generation of thrombin, leading to thrombin-induced tumorigenesis. Detailed work by Rabidazeh et al. in this issue, however, establishes that in a different tumor cell line (human prostate carcinoma cells) FGL-2 exerts direct, non-thrombin mediated angiogenic and tumorigenic activity. Using an in vitro pro-angiogenesis assay the authors showed that FGL-2 is involved in capillary sprouting that is not inhibited by hirudin, whereas FGL-2 silencing completely inhibited blood vessel formation. The role of FGL-2 in tumorigenesis was further elucidated by the authors in an in vivo mouse model of tumor development. Severe combined immunodeficiency (SCID) mice were injected with intact (wild type) or FGL-2-silenced human prostate carcinoma cell line (PC-3) clones. Injections of PC-3 with intact FGL-2 induced poorly differentiated carcinoma in all mice; malignant cells penetrated the muscle fibers and metastasized to the lung. Conversely, injection of FGL-2 silenced PC-3 clones induced tumor development only in two-thirds of the mice, moreover, tumors were significantly smaller and less aggressive, with significantly fewer blood vessels. Immunohistochemical staining revealed clearly visible FGL-2 protein in the tumor cells as well as fibroblast growth factor-2 (FGF-2). However, staining for thrombin was negative indicating a thrombin independent mechanism. In the gene-array experiments using PC-3 clones, FGF-2 was significantly downregulated by FGL-2 silencing, and this process was associated with a decrease in activation and phosphorylation of ERK1/2. These findings confirm previous reports suggesting that activation of FGF/FGF receptor signaling is sufficient to induce prostate cancer development in mouse models [19]. Results are in line with previous findings that this pathway has a well-established role in angiogenesis, wound healing and carcinogenesis [20].

The work by Rabidazeh et al provides new insight into the mechanisms underlying the proangiogenic and protumorigenic activities of FGL-2 and draws attention to a new tumor procoagulant that may serve as a link between cancer and coagulation. The importance of this topic merits further research that could serve as a basis of potential new approach to anti-tumor therapies.

\section{Conflict of Interest Statement}

The author has no conflict of interest to declare.

\section{Acknowledgement}

Z. Bagoly is supported by János Bólyai Fellowship of the Hungarian Academy of Sciences, OTKA PD111929 and Lajos Szodoray Prize of the University of Debrecen.

\section{References}

[1] Trousseau A. Phlegmasia alba dolens. Clin Med Hotel-dieu Paris 1865;3:654-712.
[2] Bouillard JB, Bouillaud S. De l'Obliteration des veines et de son influence sur la formation des hydropisies partielles: consideration sur la hydropisies passive et general. Arch Gen Med 1823;1:188-204.

[3] Blom JW, Doggen CJ, Osanto S, Rosendaal FR. Malignancies, prothrombotic mutations and the risk of venous thrombosis. JAMA 2005;293:715-22.

[4] Noble S, Pasi J. Epidemiology and pathophysiology of cancer-associated thrombosis. Br J Cancer 2010;102(Suppl. 1):S2-9.

[5] Ruf W, Disse J, Carneiro-Lobo TC, Yokota N, Schaffner F. Tissue factor and cell signalling in cancer progression and thrombosis. J Thromb Haemost 2011;9(Suppl. 1): 306-15.

[6] Gardiner C, Harrison P, Belting M, Boing A, Campello E, Carter BS, et al. Extracellular vesicles, tissue factor, cancer and thrombosis - discussion themes of the ISEV 2014 Educational Day. J Extracell Vesicles 2015;4:26901.

[7] Khorana AA, Ahrendt SA, Ryan CK, Francis CW, Hruban RH, Hu YC, et al. Tissue factor expression, angiogenesis, and thrombosis in pancreatic cancer. Clin Cancer Res 2007; $13: 2870-5$

[8] Hoffman R, Haim N, Brenner B. Cancer and thrombosis revisited. Blood Rev 2001;15: 61-7.

[9] Falanga A, Gordon SG. Isolation and characterization of cancer procoagulant: a cysteine proteinase from malignant tissue. Biochemistry 1985;24:5558-67.

[10] ten Cate H, Falanga A. Overview of the postulated mechanisms linking cancer and thrombosis. Pathophysiol Haemost Thromb 2008;36:122-30.

[11] Bezuhly M, Cullen R, Esmon CT, Morris SF, West KA, Johnston B, et al. Role of activated protein $C$ and its receptor in inhibition of tumor metastasis. Blood 2009;113: 3371-4.

[12] Horowitz NA, Blevins EA, Miller WM, Perry AR, Talmage KE, Mullins ES, et al. Thrombomodulin is a determinant of metastasis through a mechanism linked to the thrombin binding domain but not the lectin-like domain. Blood 2011;118: 2889-95.

[13] Gil-Bernabe AM, Lucotti S, Muschel RJ. Coagulation and metastasis: what does the experimental literature tell us? Br J Haematol 2013;162:433-41.

[14] Rabizadeh E, Cherny I, Lederfein D, Sherman S, Binkovsky N, Rosenblat Y, et al. The cell-membrane prothrombinase, fibrinogen-like protein 2, promotes angiogenesis and tumor development. Thromb Res 2015;136:118-24 (in this issue).

[15] Chan CW, Chan MW, Liu M, Fung L, Cole EH, Leibowitz JL, et al. Kinetic analysis of a unique direct prothrombinase, fgl2, and identification of a serine residue critical for the prothrombinase activity. J Immunol 2002;168:5170-7.

[16] Kim I, Moon SO, Koh KN, Kim H, Uhm CS, Kwak HJ, et al. Molecular cloning, expression, and characterization of angiopoietin-related protein. angiopoietin-related protein induces endothelial cell sprouting. J Biol Chem 1999;274:26523-8.

[17] Su K, Chen F, Yan WM, Zeng QL, Xu L, Xi D, et al. Fibrinogen-like protein 2/fibroleukin prothrombinase contributes to tumor hypercoagulability via IL-2 and IFN-gamma. World J Gastroenterol 2008;14:5980-9.

[18] Liu Y, Xu L, Zeng Q, Wang J, Wang M, Xi D, et al. Downregulation of FGL2/ prothrombinase delays HCCLM6 xenograft tumour growth and decreases tumour angiogenesis. Liver Int 2012;32:1585-95.

[19] Yang F, Zhang Y, Ressler SJ, Ittmann MM, Ayala GE, Dang TD, et al. FGFR1 is essential for prostate cancer progression and metastasis. Cancer Res 2013;73:3716-24.

[20] Nugent MA, Iozzo RV. Fibroblast growth factor-2. Int J Biochem Cell Biol 2000;32: 115-20.

Zsuzsa Bagoly

Division of Clinical Laboratory Sciences, University of Debrecen, Faculty of Medicine, Debrecen, Hungary

Nagyerdei krt. 98, 4032 Debrecen, Hungary. Tel.: + 36 52431956; fax: + 3652340011 E-mail address: bagoly@med.unideb.hu.

20 April 2015 